\title{
EXTENSION OF A BETA FUNCTION INEQUALITY
}

\section{PÉTER IVÁDY}

Dózsa u. 35, 9934

Hegyhátszentjakab

Hungary

e-mail: ivadyp314@t-online.hu

\begin{abstract}
In this paper, we present a beta function inequality on $[1, \infty) \times[1, \infty)$ which extends an inequality of the author. The inequality improves some beta function inequalities of Alzer and Cerone. Moreover, some elementary inequalities of two real variables are proved.
\end{abstract}

\section{Introduction}

For $x>0$, the classical gamma function $\Gamma$ and the psi function or digamma function $\Psi$ are defined as

$$
\Gamma(x)=\int_{0}^{\infty} t^{x-1} e^{-t} d t \quad \text { and } \quad \Psi(x)=\frac{\Gamma^{\prime}(x)}{\Gamma(x)} .
$$

The derivatives $\Psi^{\prime}, \Psi^{\prime \prime}, \Psi^{\prime \prime \prime}, \ldots$ are known as polygamma functions.

Closely related to the gamma function is the beta function which is the real function of two variables defined by

2010 Mathematics Subject Classification: 26D07, 33B15.

Keywords and phrases: beta function, gamma function, psi function, convex function, Hermite-Hadamard inequality.

Received July 7, 2017

(C) 2017 Scientific Advances Publishers 


$$
B(x, y)=\int_{0}^{1} t^{x-1}(1-t)^{y-1} d t, \quad x>0, y>0 .
$$

A well-known equation connecting the beta and the gamma functions

$$
B(x, y)=\frac{\Gamma(x) \Gamma(y)}{\Gamma(x+y)} .
$$

For a proof of (1.1) we refer the reader to [4], where a good reference for these functions is also given. Further literature is given in [2, 3, 6, 7, 9-14] where more references could be find.

In [12], it was shown that for all $x, y \geq 1$ the following inequality holds:

$$
B(x, y) \leq \frac{\alpha}{x y} \frac{x+y}{(x+1)(y+1)}
$$

with the best possible constant $\alpha=2$. Equality occurs in (1.2) if and only if $x=y=1$. This result is in fact an extension of the right side of the inequality of Alzer ([3], p. 738, Theorem 3.1), which reads as follows:

$\frac{1}{x y}\left(1-\alpha \frac{1-x}{1+x} \frac{1-y}{1+y}\right) \leq B(x, y) \leq \frac{1}{x y}\left(1-\beta \frac{1-x}{1+x} \frac{1-y}{1+y}\right)$, for all $x, y \in(0,1]$,

with the best possible constants $\alpha=2 / 3 \pi^{2}-4=2.57973 \ldots$ and $\beta=1$, respectively. In $[10,11]$, it was established that for all $x, y \in(0,1]$

$$
B(x, y) \leq \frac{1}{x y} \frac{x+y}{1+x y}
$$

holds. In view of (1.3), (1.4), and (1.2), it is natural to ask whether (1.4) could be true also for all $x, y \geq 1$. The main purpose of this paper is to answer this question. We show that for all $x, y \geq 1$ the inequality (1.4) remains true. 
Let us mention two related results in this direction. In ([3], p. 741, Theorem 3.4), the following result is proved. For all real numbers $x, y \geq 1$, we have

$$
\begin{aligned}
\frac{1}{\min (x, y)}\left(\frac{1}{\max (x, y)}\right)^{\min (x, y)} & \leq B(x, y) \\
& \leq \frac{1}{\min (x, y)}\left[1-\left(1-\frac{1}{\max (x, y)}\right)^{\min (x, y)}\right]
\end{aligned}
$$

Recently, Cerone ([6], p.79, Corollary 1) established for all $x, y \geq 1-$ among others - the estimations

$$
\max \left\{\frac{1}{x y^{x}}, \frac{1}{y x^{y}}\right\} \leq B(x, y) \leq \min \left\{\frac{1}{x}\left[1-\left(1-\frac{1}{y}\right)^{x}\right], \frac{1}{y}\left[1-\left(1-\frac{1}{x}\right)^{y}\right]\right\} .
$$

We will point out that (1.4) improves for all $x, y \geq 1$ (1.2), the right side of (1.5) and also the right side of (1.6).

We recall the Hermite-Hadamard inequality for a real valued convex function $f$ as follows ([15], p. 50). Let $f:[a, b] \rightarrow \mathbb{R}$ be a convex function. Then we have

$$
f\left(\frac{a+b}{2}\right) \leq \frac{1}{b-a} \int_{a}^{b} f(t) d t \leq \frac{f(a)+f(b)}{2}
$$

with $a<b$.

\section{Lemmas}

For proving our main result, we need the following lemmas. The first one can be found in ([1], Chapter 6).

Lemma 2.1. For all $x$, we have 


$$
\Gamma(x+1)=x \Gamma(x) .
$$

For all $x \neq i(i=1,2, \ldots)$, we have

$$
\begin{gathered}
\Psi(x)=-\gamma-\frac{1}{x}+x \sum_{k=1}^{\infty} \frac{1}{k(x+k)}, \quad(x \neq 0,-1,-2, \ldots), \\
\Psi^{(m)}(x)=(-1)^{m+1} m ! \sum_{i=0}^{\infty} \frac{1}{(x+i)^{m+1}}, \\
(x \neq 0,-1,-2, \ldots \text { and } m=1,2, \ldots),
\end{gathered}
$$

where $\gamma=\lim _{n \rightarrow \infty}\left(1+\frac{1}{2}+\frac{1}{3}+\ldots+\frac{1}{n}-\log n\right)=0.57721 \ldots$ is the Euler-

Mascheroni constant.

In Gordon ([8], p. 860, Theorem 4) - among other estimations - the following inequalities are given.

Lemma 2.2. For all $t>0$, we have

$$
\frac{1}{t}+\frac{1}{2 t^{2}}+\frac{1}{6\left(t+\frac{1}{14}\right)^{3}}<\Psi^{\prime}(t)<\frac{1}{t}+\frac{1}{2 t^{2}}+\frac{1}{6 t^{3}}
$$

Lemma 2.3. Let $x, y \geq 1$, then we have

$$
p(x, y):=2 x y^{3}-y^{3}+6 x^{2} y^{2}-y^{2}+4 x^{3} y-2 x y+y-1>0 .
$$

Proof. It is clear that $p(1,1)=8$. Differentiation gives

$$
\frac{\partial p(x, y)}{\partial x}=2 y^{3}+12 x y^{2}+12 x^{2} y-2 y>0,
$$

therefore $p(x, y)$ is strictly increasing in $x$ and we have

$$
p(x, y) \geq p(1, y)=y^{3}+5 y^{2}+3 y-1>0 .
$$




\section{Main Result}

Now we give the main result of this paper.

Theorem. For all real numbers $x \geq 1$ and $y \geq 1$, we have

$$
B(x, y) \leq \frac{1}{x y} \frac{x+y}{1+x y}
$$

and equality occurs in (3.1) if and only if $x=1$ or $y=1$. For $x \geq 1$ and $0<y \leq 1$ or $y \geq 1$ and $0<x \leq 1$, (3.1) is reversed.

Proof. To establish (3.1), let

$$
F(x, y):=\log \frac{\Gamma(x+1) \Gamma(y+1)}{\Gamma(x+y)}-\log \frac{x+y}{1+x y} .
$$

Without loss of any generality, we can assume that $1 \leq x<y<\infty$. Building the derivative, we get

$$
\frac{\partial F(x, y)}{\partial x}=\frac{(y+1)(y-1)}{(x+y)(1+x y)}-\Psi(x+y)+\Psi(x+1) .
$$

In fact we have $\partial F(x, y) / \partial x<0$. Since $\Psi^{\prime}(t)$ is convex [cf. (2.3)] and applying the Hermite-Hadamard inequality (1.7), we find

$$
(y-1) \Psi^{\prime}\left(x+\frac{y+1}{2}\right) \leq \int_{x+1}^{x+y} \Psi^{\prime}(t) d t=\Psi(x+y)-\Psi(x+1) .
$$

In view of (3.2), (3.3) and in conjunction with the left side of (2.4), if we have

$$
\begin{aligned}
\frac{(y+1)(y-1)}{(x+y)(1+x y)} & \leq(y-1)\left[\frac{1}{x+\frac{y+1}{2}}+\frac{1}{2\left(x+\frac{y+1}{2}\right)^{2}}\right] \leq(y-1) \Psi^{\prime}\left(x+\frac{y+1}{2}\right) \\
& \leq \Psi(x+y)-\Psi(x+1)
\end{aligned}
$$


then we imply $\partial F(x, y) / \partial x<0$. Now we obtain after some computations

$$
\begin{aligned}
\Delta(x, y) & :=\frac{(y+1)}{(x+y)(1+x y)}-\left[\frac{1}{x+\frac{y+1}{2}}+\frac{1}{2\left(x+\frac{y+1}{2}\right)^{2}}\right] \\
& =-\frac{P(x, y)}{(x+y)(1+x y)(1+2 x+y)^{2}}
\end{aligned}
$$

where

$$
P(x, y):=2 x y^{3}-y^{3}+6 x^{2} y^{2}-y^{2}+4 x^{3} y-2 x y+y-1 .
$$

According to Lemma 2.3, we claim that $P(x, y)>0$ for all $x, y \geq 1$. Therefore we get $\Delta(x, y)<0$, and $\partial F(x, y) / \partial x<0$ which means $F(x, y)$ is strictly decreasing in $x$, i.e., $F(x, y) \leq F(1, y)=0$ and the Theorem is proved.

The reversed inequality could be prove by a similar argument, using the Hermite-Hadamard inequality (1.7) again, so we omit the details.

\section{Concluding Remarks}

Now we show that (3.1) improves (1.2), (1.5), and (1.6) as announced in the Introduction. It is clear that the following relations hold:

Corollary 4.1. Let $x, y \geq 1$, then we have

$$
\frac{x+y}{1+x y} \leq 1-\frac{x-1}{\sqrt{x^{2}+1}} \frac{y-1}{\sqrt{y^{2}+1}} \leq \frac{2(x+y)}{(x+1)(y+1)} .
$$

Equality in the first inequality of (4.1) holds if and only if $x=1$ or $y=1$ or $x=y$. Whilst equality for the second inequality in (4.1) holds if and only if $x=1$ or $y=1$.

The next corollary shows that (3.1) improves (1.5). 
Corollary 4.2. For all real numbers $x, y \geq 1$, we have

$$
\frac{1}{x y} \frac{x+y}{1+x y} \leq \frac{1}{\min (x, y)}\left[1-\left(1-\frac{1}{\max (x, y)}\right)^{\min (x, y)}\right],
$$

with equality if and only if $x=y=1$.

Proof. Suppose that $1<x<y<\infty$. Let

$$
H(x, y):=x \log \left(1-\frac{1}{y}\right)-\log \left(1-\frac{x+y}{y(1+x y)}\right) .
$$

Differentiation gives

$$
\frac{\partial H(x, y)}{\partial x}=\log \left(1-\frac{1}{y}\right)-\frac{1}{x(1+x y)}<0
$$

i.e., $H(x, y)$ is strictly decreasing in $x$ thus we have $H(x, y) \leq H(1, y)=0$ which we had to prove.

Finally, we show that (3.1) also improves (1.6).

Corollary 4.3. For all real numbers $x, y \geq 1$, we have

$$
\frac{1}{x y} \frac{x+y}{1+x y} \leq \min \left\{\frac{1}{x}\left[1-\left(1-\frac{1}{y}\right)^{x}\right], \frac{1}{y}\left[1-\left(1-\frac{1}{x}\right)^{y}\right]\right\},
$$

with equality if and only if $x=y=1$.

Proof. We prove only

$$
\frac{1}{x y} \frac{x+y}{1+x y} \leq \frac{1}{x}\left[1-\left(1-\frac{1}{y}\right)^{x}\right]
$$

because the other one could be proved similarly. We define

$$
H_{1}(x, y):=x \log \left(1-\frac{1}{y}\right)-\log \left(\frac{x(y+1)(y-1)}{y(1+x y)}\right) .
$$

Then we get 


$$
\frac{\partial H_{1}(x, y)}{\partial x}=\log \left(1-\frac{1}{y}\right)-\frac{1}{x(1+x y)}<0
$$

therefore $H_{1}(x, y)$ is strictly decreasing in $x$ so we conclude $H_{1}(x, y) \leq$ $H_{1}(1, y)=0$.

\section{References}

[1] M. Abramowitz and I. A. Stegun, (Editors), Handbook of Mathematical Functions with Formulas Graphs and Mathematical Tables, Dover, New York, 1965.

[2] H. Alzer, Sharp inequalities for the beta function, Indag. Math. 12 (2001), 15-21.

[3] H. Alzer, Some beta function inequalities, Proc. of the Royal Soc. of Edinburgh 133A (2003), 731-745.

[4] G. D. Anderson, M. K. Vamanamurthy and M. Vuorinen, Conformal Invariants Inequalities and Quasiconformal Maps, Wiley, New York, 1997.

[5] G. E. Andrews, R. Askey and R. Roy, Special Functions, Cambridge University Press, Cambridge, 1999.

[6] P. Cerone, Special functions: Approximations and bounds, Appl. Anal. Discrete Math. 1 (2007), 72-91.

[7] S. S. Dragomir, R. P. Agarwal and N. S. Barnett, Inequalities for beta and gamma functions via some classical and new integral inequalities, J. Inequal. Appl. 5 (2000), 103-165.

[8] L. Gordon, A stochastic approach to the gamma function, Amer. Math. Monthly 101 (1994), 858-865.

[9] L. Grenié and G. Molteni, Inequalities for the beta function, J. Math. Inequal. Appl. 18 (2015), 1427-1442.

[10] P. Ivády, On a beta function inequality, J. Math. Inequal. 6(3) (2012), 333-341.

[11] P. Ivády, Corrigendum to: On a beta function inequality, J. Math. Inequal. 10(2) (2016), 597-601.

[12] P. Ivády, On a beta function inequality II, J. Math. Sciences: Adv. and Appl. 22 (2013), 27-37.

[13] G. Lochs, Abschätzung spezieller Werte der unvollständigen Betafunktion, Anz. Österr Akad. Wissensch 123 (1986), 59-63.

[14] D. S. Mitrinović, Analytic Inequalities, Springer, 1970.

[15] C. Niculescu and L. E. Persson, Convex Functions and their Applications, Springer, 2006. 